\title{
One-dimensional linear analysis of the compound jet
}

\author{
By ANGEL SANZ AND JOSÉ MESEGUER
}

The stability of an infinitely long compound liquid column is analysed by using a one-dimensional inviscid slice model. Results obtained from this one-dimensional linear analysis are applicable to the study of compound capillary jets, which are used in the ink-jet printing technique. Stability limits and the breaking regimes of such fluid configurations are established, and, whenever possible, theoretical results are compared with experimental ones.

\section{Introduction}

During the last two decades fine jets of ink have been increasingly used for printing purposes. Developments in ink-jet technology have motivated numerous scientists to investigate the details of the breaking of capillary jets emerging from nozzles. A review of the state of the art in this field at the end of the 1970s can be found in Bogy (1979). This review should be complemented by some subsequent significant papers: Chaudhary \& Redekopp (1980), Bogy (1981), Entov \& Yarin (1984), among others.

Recently a new ink-jet printing method (the compound jet) has been developed at the Lund Institute of Technology in Sweden (Hermanrud \& Hertz 1979; Hermanrud 1981 ; Hertz \& Hermanrud 1983). A compound jet, as sketched in figure 1, is generated as follows. A fine nozzle is submerged below the surface of a stationary fluid (ink). By forcing a suitable 'inner' fluid through this nozzle under high pressure a liquid-into-liquid jet is generated in the stationary fluid (the 'outer' fluid). Owing to viscous forces the outer fluid will be accelerated by the inner fluid close to the interface between the two fluids. Therefore when the jet emerges into the air it consists of a cylindrical core of fluid (the inner jet) surrounded by a concentric layer of different fluid (the outer jet), both travelling at essentially the same speed.

Published papers concerning compound jets deal mainly with experimental work. Theoretical developments in this field have been limited to rough analyses which are quite far from the sophisticated studies on single capillary jets. However, regarding the partial similarity between compound jets and compound liquid columns, the work of Bauer (1982) and Sanz (1983) should be noted. In the first paper the linear stability of an infinitely long liquid column is analysed by using a three-dimensional model based on previous work of Tomotika (1935), whereas the second is devoted to the study of liquid bridges surrounded by another liquid.

In the present paper the linear stability of the compound jet is studied through a one-dimensional inviseid model which is a generalization of that of Lee (1974) for single capillary jets. This model has been selected instead of more complicated one-dimensional models (Weber 1931 ; Green 1976; Entov \& Yarin 1984) because, in spite of its relative simplicity, the results obtained are in agreement with experimental evidence, both in the case of single capillary jets (Pimbley \& Lee 1977) and in the 
case of slender liquid bridges (Sanz 1983; Meseguer \& Sanz 1985). Amongst the different types of instability appearing in a compound jet (Hertz \& Hermanrud 1983) we analyse capillary instability only, which is the most interesting in ink-jet printing applications in order to predict the size of the resulting drops after the jet breakup. The remaining instabilities (sinuous and varicose instability) are outside the scope of this paper, and they seem to be more easily dealt with by means of an experimental approach rather than a theoretical one. We assume that viscosity effects (if low-viscosity liquids are involved) are important only in the nozzle region, where the compound jet is set up, and that these effects can be neglected in the study of capillary instability, which appears in a region far from the nozzle. A similar hypothesis is used in, for instance, the analysis of the response of a boundary layer to a small disturbance: it is assumed not to be affected by the viscosity of the fluid, even though viscosity was of course essential for the setting up of the velocity distribution in the undisturbed boundary layer.

Finally, theoretical results have been compared with available experimental results (Hertz \& Hermanrud 1983) and reasonable agreement has been found.

\section{General equations for the one-dimensional compound jet}

According to Bogy (1979), studies on capillary-jet instability could be classified in two main categories: temporal-instability and spatial-instability analyses. Keller, Rubinow \& Tu (1973) showed the suitabilit; of spatial instability analyses for describing the behaviour of capillary jets. These authors also verified the agreement between results obtained from the temporal or the spatial approach when the jet velocity is much higher than the capillary velocity, which is the case in ink-jet printing. Therefore in this paper we analyse temporal instability, since its formulation is simpler than that of spatial instability. To perform this study it must be assumed that the reference system is moving with a velocity equal to the mean jet velocity, so that equations of motion become similar to that of a compound liquid column.

Let us consider a compound jet as sketched in figure 1 , and concentrate on the region far enough from the nozzle. To carry out the analysis of this liquid configuration several assumptions are introduced:

(a) internal movement in the compound jet is due only to capillary-pressure gradients generated by the deformation of interfaces;

(b) the dynamies of the compound jet is not affected by the surrounding air;

(c) since only axisymmetric configurations are considered, the problem is independent of the azimuthal coordinate;

$(d)$ both liquids are inviseid, $\uparrow$ with constant and uniform properties (density and surface tension);

(e) in each of the liquids the axial velocity, as well as the pressure, depends on the axial coordinate and the time, but not on the radial coordinate.

This last hypothesis, which is the more drastic assumption introduced by the

$\uparrow$ The effect of viscosity within the one-dimensional model used could be accounted for through an unsteady boundary layer at the interface between the two liquids, to accommodate the shear stresses and the velocity jump. The thickness of this boundary layer would be $\delta \sim(\nu / \tau)^{\frac{1}{2}}$ (Schlichting 1960 ), where $\tau$ is a characteristic dimensional growth factor and $\nu$ the kinematic viscosity (almost the same in both liquids). As we shall see, $\tau \sim\left(\rho^{\circ} / \sigma^{\circ} R^{\circ 3}\right)^{\frac{1}{2}}$, where $\sigma^{\circ}$, $\rho^{\circ}$ and $R^{\circ}$ are respectively the surface tension, density and radius of the outer jet. Thus $\delta / R^{\circ} \sim\left(v^{2} \rho^{\circ} / \sigma^{\circ} R^{\circ}\right)^{\frac{1}{4}}=0.16$, for the values quoted in Hertz \& Hermanrud (1983): $\sigma^{0}=2 \times 10^{-2} \mathrm{~N} \mathrm{~m}^{-1}, \quad \rho^{0}=10^{3} \mathrm{~kg} \mathrm{~m}^{-3}$, $R^{\circ}=3 \times 10^{-4} \mathrm{~m}$ and $\nu=2 \times 10^{-6} \mathrm{~m}^{2} \mathrm{~s}^{-1}$. 


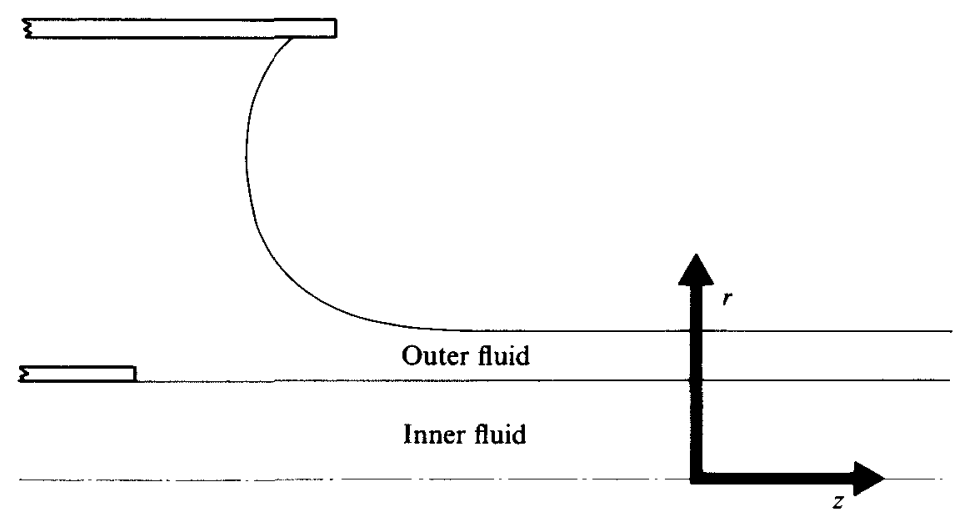

Figure 1. Geometry and coordinate system for the compound jet.

one-dimensional model, is justified in the case of compound jets because perturbation wavelengths involved in jet breaking are generally larger than the jet radius (Meseguer 1983; Sanz 1983).

Under these hypotheses the equations of motion are drastically reduced. The radial momentum equation becomes uncoupled and the problem formulation reduces to the continuity equation and axial momentum equation, plus suitable initial and boundary conditions. To generate the equation set for the compound jet the procedure is similar to that described in Lee (1974), Meseguer (1983) and Sanz (1983). In the following $F^{j}$ stands for the interface radius, $W^{j}$ for the axial velocity and $P^{j}$ for pressure; $\rho^{j}$ and $\sigma^{j}$ are density and interfacial tension respectively. The superscript $j$ denotes the liquid (i for inner and o for outer, see figure 1), whereas the subscripts $t$ and $z$ indicate time and spatial derivatives respectively. The equations for the compound jet are as follows.

(a) Inner jet:

(a 1) continuity equation

$$
\left(F^{\mathrm{i} 2}\right)_{t}+\left(W^{\mathrm{i}} F^{\mathrm{i} 2}\right)_{z}=0
$$

(a 2) axial momentum equation

$$
W_{t}^{\mathrm{i}}+W^{\mathrm{i}} W_{z}^{\mathrm{i}}=\frac{-P_{z}^{\mathrm{i}}}{\rho^{i}}
$$

(b) Outer jet:

(b 1$)$ continuity equation

$$
\left(F^{\mathrm{o} 2}-F^{\mathrm{i} 2}\right)_{t}+\left[W^{\mathrm{o}}\left(F^{\mathrm{o} 2}-F^{\mathrm{i} 2}\right)\right]_{z}=0,
$$

(b 2) axial momentum equation

$$
W_{t}^{\mathrm{o}}+W^{\mathrm{o}} W_{z}^{\mathrm{o}}=\frac{-P_{z}^{\mathrm{o}}}{\rho^{\mathrm{o}}} .
$$

$P^{\mathrm{i}}, P^{\mathrm{o}}$ and the external pressure $P^{\mathrm{e}}$ are related through

$$
\begin{gathered}
P^{\mathrm{i}}-P^{o}=\sigma^{\mathrm{i}} \mathbb{P}\left(F^{\mathrm{i}}\right), \quad P^{\mathrm{o}}-P^{\mathrm{e}}=\sigma^{o} \mathbb{P}\left(F^{\mathrm{o}}\right), \\
\mathbb{P}\left(F^{j}\right)=\left[1+\left(F_{z}^{j}\right)^{2}\right]^{-\frac{3}{2}}\left\{\frac{1+\left(F_{z}^{j}\right)^{2}}{F^{j}}-F_{z z}^{j}\right\} .
\end{gathered}
$$


To put these equations in non-dimensional form, we take as reference the properties of the outer liquid: undisturbed interface radius $R^{o}$, density $\rho^{\circ}$ and surface tension $\sigma^{\circ}$. To that purpose, we introduce

$$
F^{j}=R^{0} \bar{F}^{j}, \quad z=R^{o} \bar{z}, \quad t=\left(\frac{\rho^{o} R^{03}}{\sigma^{0}}\right)^{\frac{1}{2}} \bar{t}, \quad W^{j}=\left(\frac{\sigma^{\circ}}{R^{\mathrm{o}} \rho^{\mathrm{o}}}\right)^{\frac{1}{2}} \bar{W}^{j}, \quad P^{j}=\frac{\sigma^{\mathrm{o}}}{R^{\mathrm{o}}} \bar{P}^{j},
$$

where the overbarred quantities are dimensionless. We introduce also the parameters

$$
R=\frac{R^{\mathrm{i}}}{R^{\mathrm{0}}}, \quad \rho=\frac{\rho^{\mathrm{i}}}{\rho^{\mathrm{0}}}, \quad \sigma=\frac{\sigma^{\mathrm{i}}}{\sigma^{\mathrm{0}}},
$$

$R^{\mathrm{i}}$ being the undisturbed inner-jet radius. Then $(2.1)-(2.6)$ yield the following dimensionless equations (with the overbars dropped from now on):

$$
\begin{gathered}
\left(F^{\mathrm{i} 2}\right)_{t}+\left(W^{\mathrm{i}} F^{\mathrm{i} 2}\right)_{z}=0, \\
W_{t}^{\mathrm{i}}+W^{\mathrm{i}} W_{z}^{\mathrm{i}}=\frac{-P_{z}^{\mathrm{i}}}{\rho}, \\
\left(F^{\mathrm{o} 2}-F^{\mathrm{i} 2}\right)_{t}+\left[W^{\mathrm{o}}\left(F^{\mathrm{o} 2}-F^{\mathrm{i} 2}\right)\right]_{z}=0, \\
W_{t}^{\mathrm{o}}+W^{\mathrm{o}} W_{z}^{\mathrm{o}}=-P_{z}^{o}, \\
P^{\mathrm{i}}-P^{\mathrm{o}}=\sigma \mathbb{P}\left(F^{\mathrm{i}}\right), \\
P^{\mathrm{o}}-P^{\mathrm{e}}=\mathbb{P}\left(F^{\mathrm{o}}\right), \\
\mathbb{P}\left(F^{j}\right)=\left[1+\left(F_{z}^{j}\right)^{2}\right]^{-\frac{3}{2}}\left\{\frac{1+\left(F_{z}^{j}\right)^{2}}{F^{j}}-F_{z z}^{j}\right\} .
\end{gathered}
$$

Note that $F^{j}, W^{j}, t$, etc. are now dimensionless variables, and that the undisturbed interface shapes are $F^{\mathrm{i}}=R$ and $F^{\circ}=1$.

In conclusion, the problem formulation consists of four nonlinear differential equations, which, once initial and boundary conditions are fixed, allow the calculation of $F^{\mathrm{i}}, F^{\circ}, W^{\mathrm{i}}$ and $W^{\mathrm{o}}$.

\section{Linear analysis}

Let $\epsilon$ be a small parameter, measuring, for instance, the initial deviation of the outer interface shape from the cylindrical one. If $\epsilon$ is small enough, ignoring $\epsilon^{2}$ terms, the variables involved in the problem may be rewritten as

$$
F^{\mathrm{i}}=R+\epsilon f^{\mathrm{i}}, \quad F^{\mathrm{o}}=1+\epsilon f^{\mathrm{o}}, \quad W^{\mathrm{i}}=\epsilon w^{\mathrm{i}}, \quad W^{\mathrm{o}}=\epsilon w^{\mathrm{o}}, \quad \text { etc. }
$$

$\mathbb{P}\left(F^{j}\right)$ now takes the form

$$
\begin{gathered}
\mathbb{P}\left(F^{\mathrm{i}}\right)=\frac{1}{R}-\epsilon\left(f_{z z}^{\mathrm{i}}+\frac{f^{\mathrm{i}}}{R^{2}}\right), \\
\mathbb{P}\left(F^{\mathrm{o}}\right)=1-\epsilon\left(f_{z z}^{o}+f^{\mathrm{o}}\right) .
\end{gathered}
$$

After substituting these expressions in (2.9)-(2.14) the following linearized problem is obtained:

$$
\begin{gathered}
2 f_{t}^{\mathrm{i}}+R w_{z}^{\mathrm{i}}=0, \\
\rho w_{t}^{\mathrm{i}}=\sigma\left(f_{z z z}^{\mathrm{i}}+\frac{f_{z}^{\mathrm{i}}}{R^{2}}\right)+f_{z z z}^{\mathrm{o}}+f_{z}^{o}, \\
2 f_{t}^{\mathrm{o}}-2 R f_{t}^{\mathrm{i}}+w_{z}^{\mathrm{o}}\left(1-R^{2}\right)=0, \\
w_{t}^{\mathrm{o}}=f_{z z z}^{\mathrm{o}}+f_{z}^{\mathrm{o}} .
\end{gathered}
$$




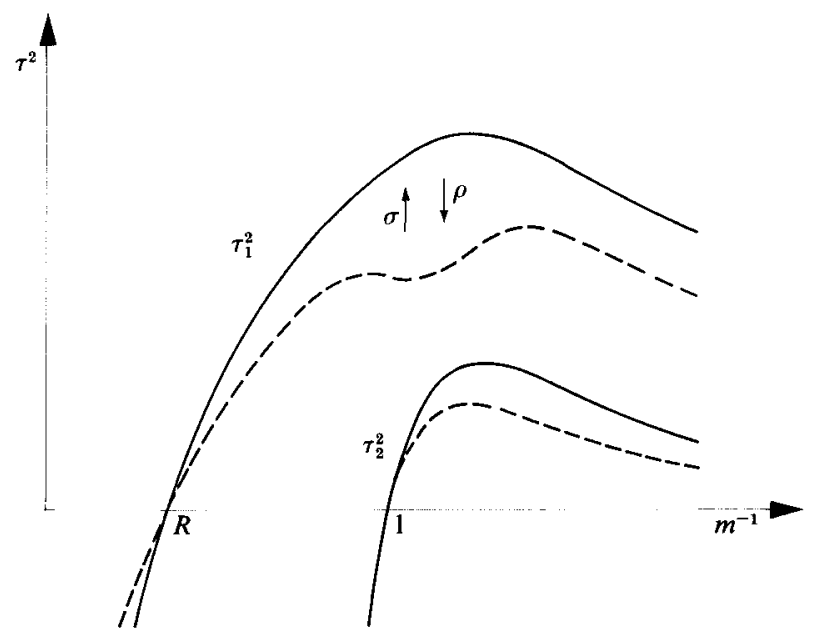

Figure 2. Qualitative influence of $\sigma$ (inner to outer surface-tension ratio) and $\rho$ (inner to outer density ratio) on the variation with the inverse wavenumber $m^{-1}$ of the roots $\tau_{1}^{2}$ and $\tau_{2}^{2}$ of (3.10). Arrows indicate the variation of the root curves as $\sigma$ or $\rho$ increase.

This system of four equations with four unknown functions can be reduced to one single equation. Differentiation of (3.4) with respect to time and of (3.5) with respect to $z$ allow us to eliminate $w^{\mathrm{i}}$ between these two equations. In a similar way $w^{\circ}$ may be eliminated between (3.6) and (3.7), and eliminating $f^{i}$ between the two resulting equations yiclds

$$
\begin{aligned}
\frac{2 \rho}{R^{2}} \frac{\partial^{4} f^{\circ}}{\partial t^{4}}+\left(1-\rho+\frac{\rho}{R^{2}}+\frac{\sigma}{R}\right) & \frac{\partial^{6} f^{\circ}}{\partial t^{2} \partial z^{4}}+\left(1-\rho+\frac{\rho}{R^{2}}+\frac{\sigma}{R^{3}}\right) \frac{\partial^{4} f^{o}}{\partial t^{2} \partial z^{2}} \\
& +\frac{\sigma\left(1-R^{2}\right)}{2 R}\left[\frac{\partial^{8} f^{o}}{\partial z^{8}}+\left(1+\frac{1}{R^{2}}\right) \frac{\partial^{6} f^{o}}{\partial z^{6}}+\frac{1}{R^{2}} \frac{\partial^{4} f^{\circ}}{\partial z^{4}}\right]=0 .
\end{aligned}
$$

To analyse temporal instabilities we try solutions like

$$
f^{0}=C \mathbf{e}^{\tau t+\mathbf{i} m z}
$$

so that the following dispersion equation results:

$$
\begin{aligned}
\frac{2 \rho}{R^{2}} \tau^{4}-\left[\left(1+\rho \frac{1-R^{2}}{R^{2}}\right)\left(1-m^{2}\right)+\frac{\sigma}{R}(\right. & \left.\left.\frac{1}{R^{2}}-m^{2}\right)\right] m^{2} \tau^{2} \\
& +\frac{\sigma\left(1-R^{2}\right)}{2 R} m^{4}\left(1-m^{2}\right)\left(\frac{1}{R^{2}}-m^{2}\right)=0 .
\end{aligned}
$$

For each value of the wavenumber $m$ there are four roots $\pm \tau_{1}, \pm \tau_{2}$ which determine four superposed evolutions. As can easily be demonstrated, the discriminant of (3.10) is always positive, hence $\tau_{1}^{2}$ and $\tau_{2}^{2}$ are real numbers, and time evolutions of the compound jet are oseillatory or grow exponentially, as one would expect from an inviscid model.

In figure 2 the variation with the inverse wavenumber $m^{-1}$ of the growth factors $\tau_{1}^{2}$ and $\tau_{2}^{2}$ is shown. If the dimensionless perturbation wavelength $\Lambda=2 \pi m^{-1}$ is smaller than $2 \pi R$ both roots are negative; this means an oscillatory motion, the compound jet is stable under such short-wavelength perturbations. If $\Lambda$ is large enough $(\Lambda>2 \pi)$ both roots are positive: the compound jet breaks. There is a middle region $(2 \pi R<A<2 \pi)$ in which one root is positive and the other negative, which corresponds 

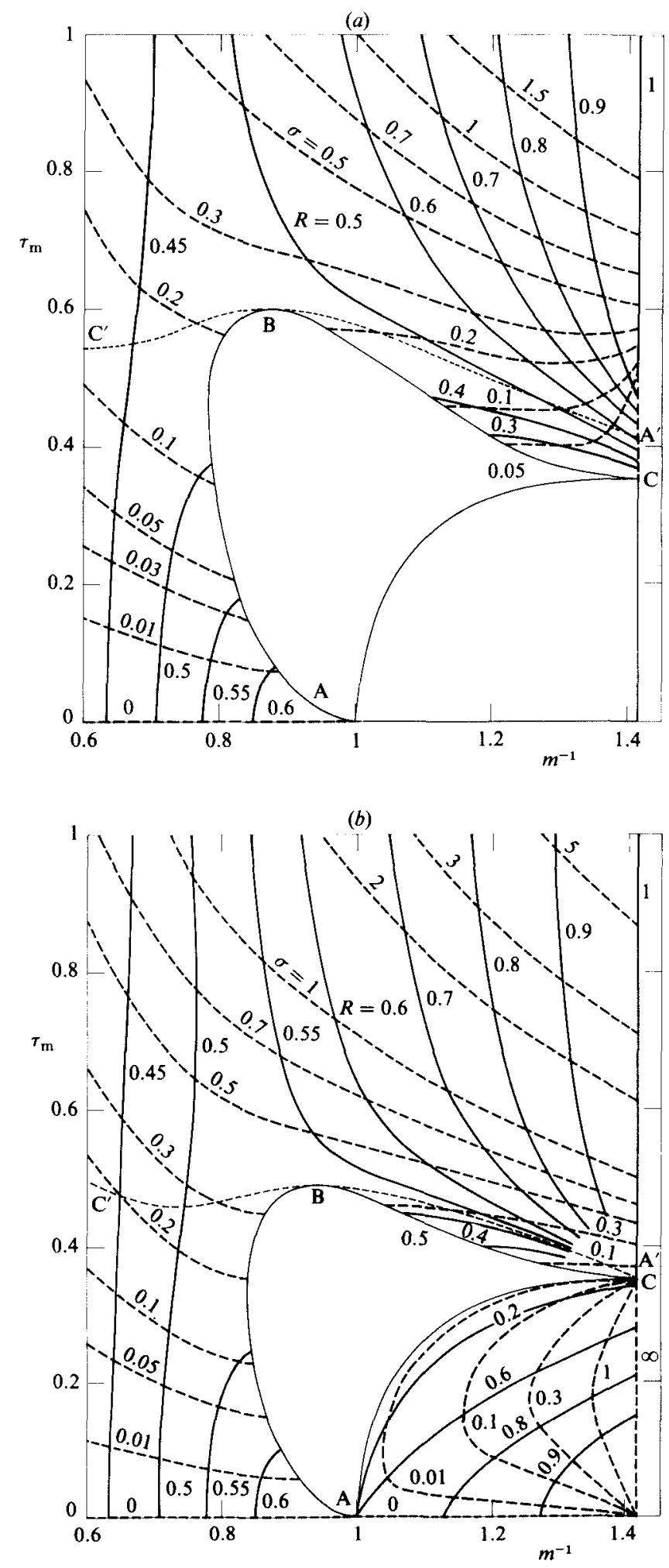

Figure 3(a,b). For description see facing page. 

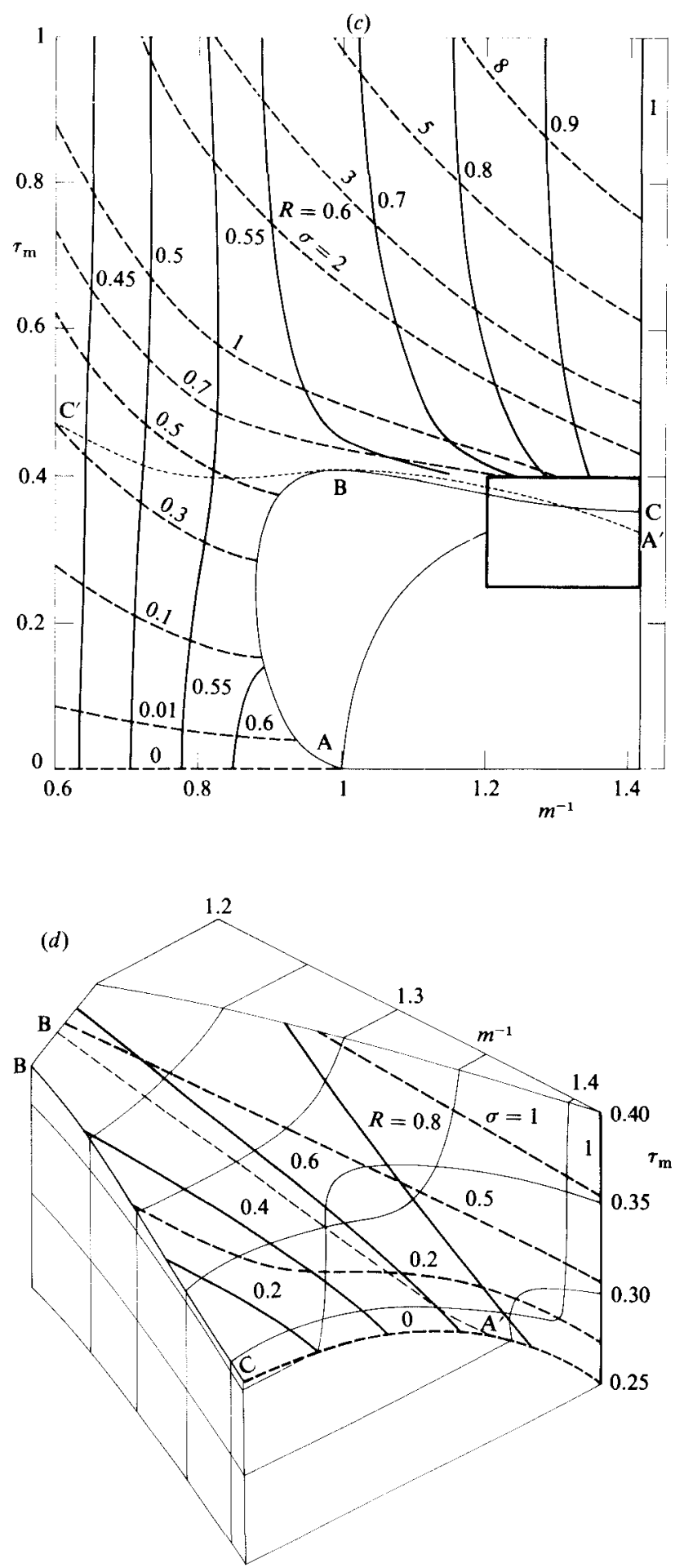

FIgURE 3. Maximum growth factor $\tau_{m}$ versus inverse wavenumber $m^{-1}$ of eompound jets with inner to outer density ratios $\rho=0.5(a), 1(b)$ and $2(c)$. Numbers on the curves indicate the values of the inner to outer radius ratio $R$ (solid lines) and the inner to outer surface-tension ratio $\sigma$ (dashed lines). Details on the box outlined in $(c)$ are shown in $(d)$. 


\begin{tabular}{ccccc} 
& \multicolumn{3}{c}{ Optimum breakup condition } & \\
Case & $\tau_{\mathrm{m}}^{2}$ & $m^{-1}$ & Comments \\
\cline { 2 - 3 }$\sigma \rightarrow \infty$ & $\infty$ & $\sqrt{ } 2 R$ & $1 / R$ & \\
$\sigma=0$ & $\frac{1}{8}\left(1+R^{2} \frac{1-\rho}{\rho}\right)$ & $\sqrt{ } 2$ & $\frac{R}{\rho+R^{2}(1-\rho)}$ & \\
$\sigma=0$ & 0 & $\sqrt{ } 2 R$ & $\infty$ & $0 \leqslant R \leqslant 1 / \sqrt{ } 2$ \\
$R=1$ & $\frac{1+\sigma}{8 \rho}$ & $\sqrt{ } 2$ & 1 & single jet \\
$R=0$ & $\frac{1}{8}$ & $\sqrt{ } 2$ & $\infty$ & single jet \\
$\rho \rightarrow \infty$ & & $\sqrt{ } 2$ & 0 & solid-core jet
\end{tabular}

TABLE 1

also to breaking evolutions. Therefore there is a stability limit $(A=2 \pi R)$ depending only on the value of $R$, and two breaking regimes.

Within time-stability studies, it is generally accepted that capillary jets break owing to the fastest-growing spatial harmonic (Lee 1974; Bogy 1978). Therefore the optimum condition for breaking means maximum $\tau$. As can be seen in figure 2 , the upper branch $\tau_{1}^{2}$ presents one or two maxima, depending on the values of $R, \rho$ and $\sigma$, whereas in the lower branch $\tau_{2}^{2}$ there is one maximum only. From the point of view of instability, the lower-branch roots are irrelevant, because, for each value of the wavenumber $m$, the upper-branch roots are always larger.

The variation with $m^{-1}$ of the maximum growth factor $\tau_{\mathrm{m}}$ for several values of $R$, $\rho$ and $\sigma$ is plotted in figure 3 (in figure $3(b)$ maxima of the lower-branch roots have also been represented). Analytical expressions of $\tau_{m}$ for some extreme cases are shown in table 1. Concerning upper-branch maxima represented in figure 3 , there is a middle region where no maxima exist. For instance, in the case $\rho=1, R=0.5$ and $\sigma \rightarrow 0$ (figure $3 b$ ) there is a maximum at $m^{-1} \approx 0.7$ and a second one at $m^{-1} \approx 1.4$; when $\sigma$ increases, the first maximum increases, whereas the second vanishes at $\sigma \approx 0.26$ (at this point the fading maximum becomes an inflexion point, see figure 2). Each point $(R, \sigma)$ of the inflexion curve $\mathrm{ABC}$ defines the points of a second curve $\mathrm{A}^{\prime} \mathrm{BC}^{\prime}$ corresponding to the other maximum, so that eurves $\mathrm{ABC}$ and $\mathrm{A}^{\prime} \mathrm{BC}^{\prime}$ split the maximum-roots region into two zones. Therefore two possibilities appear.

(I) Above $\mathrm{A}^{\prime} \mathrm{BC}^{\prime}$ there is one maximum only, which determines the optimum condition for breakup. When $\rho=1, R \sim 1$ and $\sigma \rightarrow 0$ (the compound jet becomes a single jet) the optimum condition for breakup is reached at $m^{-1}=\sqrt{ } 2$, with a maximum growth factor $\tau_{\mathrm{m}}=1 / \sqrt{ } 8$, which are the same values as those calculated by Lee (1974) for the single jet. If $R>\sqrt{ } 2(R=\sqrt{ } 2$ means both inner and outer jets have the same cross-sectional area) $\tau_{\mathrm{m}}$ occurs at $m^{-1}>1$ regardless of the value of $\sigma$; this seems to indicate that the breaking process will be determined by the outer interface. In contrast, when $R<\sqrt{ } 2$ the maximum growth factor occurs at $m^{-1} \lesssim 1$, and the breaking process is mainly driven by the inner interface.

(II) Between $\mathrm{ABC}$ and $\mathrm{A}^{\prime} \mathrm{BC}^{\prime}$, for each pair $(R, \sigma)$ there are two relative maxima. Since the inflexion curve $\mathrm{ABC}$ vanishes at $m^{-1}=1$ when $R=\sqrt{ } 2$, points of the two-maxima region can only be reached from compound-jet configurations having $R<\sqrt{ } 2$ and low values of $\sigma$. Of these two optimum breakup conditions, that having the highest value of $\tau_{\mathrm{m}}$ will develop faster and will become dominant in the breaking process. When $\sigma \rightarrow 0$ the highest $\tau_{m}$ occurs at $m^{-1} \approx \sqrt{ } 2$; but, as $\sigma$ increases, both 




Figure 4. Minimum jet radius $F_{\mathrm{m}}^{j}$ versus time $t$. Inner-jet breaking time $t_{\mathrm{b}}^{\mathrm{i}}$ is obtained when its minimum radius $F_{\mathrm{m}}^{\mathrm{i}}$ vanishes. In the outer-jet case there are two possibilities, and breaking time $t_{\mathrm{b}}^{\mathrm{o}}$ is reached when its minimum radius $F_{\mathrm{m}}^{\mathrm{o}}$ vanishes $(a)$ or when the outer interface reaches the inner one $(b)$.

values of $\tau_{m}$ become of the same order, and, in general, determination of which one is dominant would require a more detailed analysis.

When $\rho \neq 1$ the behaviour of the compound jet is similar to that described above, the main differences appearing close to $m^{-1}=\sqrt{ } \mathbf{2}$, where, even when $\sigma \rightarrow 0$, the compound jet does not behave as a single jet. In this case, $\tau_{\mathrm{m}}$ increases as $R$ grows when $\rho<1$, and the contrary occurs when $\rho>1$. This behaviour can be explained by inner-jet inertial effects, which cause time evolution to be retarded as the density ratio $\rho$ grows.

\section{Breaking regimes}

To discuss the possible breaking regimes of the compound jet, we introduce two new parameters: the amplification $A=f^{\mathrm{i}} / f^{\circ}$, defined as the ratio of the maximum (or minimum) inner-interface deformation to the maximum (or minimum) outerinterface deformation, and the linear breaking time $t_{\mathrm{b}}^{j}$.

The amplification is calculated by eliminating $w^{\circ}$ between (3.6) and (3.7) as explained above. Thus the following relationship is obtained:

$$
R f_{t t}^{\mathrm{i}}=f_{t t}^{\mathrm{o}}+\frac{1}{2}\left(1-R^{2}\right)\left(f_{z z z z}^{\mathrm{o}}+f_{z z}^{\mathrm{o}}\right) ;
$$

therefore, for each pair $(\tau, m)$,

$$
A=\frac{1}{R}\left[1+\frac{1-R^{2}}{2 \tau^{2}}\left(m^{4}-m^{2}\right)\right] .
$$

Concerning $t_{\mathrm{b}}^{j}$, according to (3.1), (3.9) and (4.2), the time evolutions of the minimum inner and outer jet radii are respectively

$$
F_{\mathrm{m}}^{\mathrm{i}}=R-C \epsilon A \mathrm{e}^{\tau t}, \quad F_{\mathrm{m}}^{\mathrm{o}}=1-C \epsilon \mathrm{e}^{\tau t} .
$$



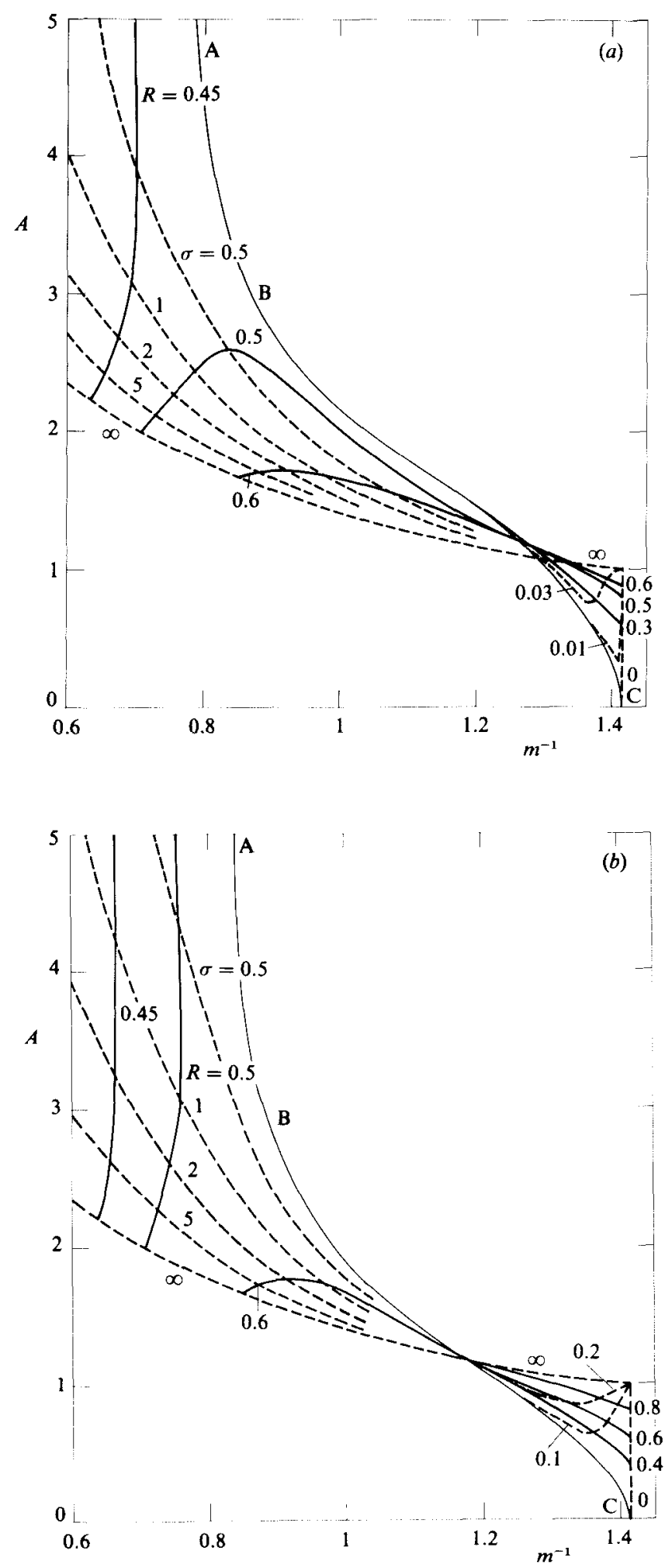

Figura $5(a, b)$. For description see facing page. 


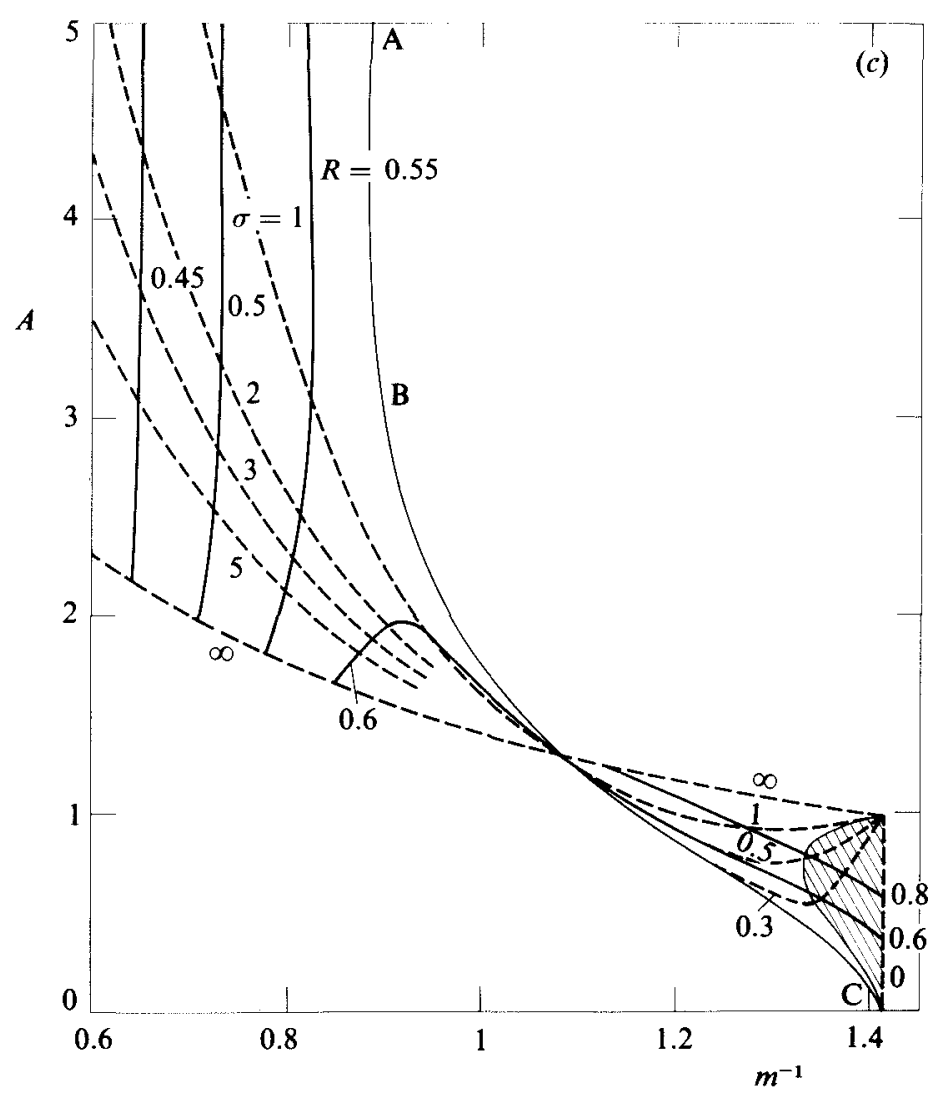

Figure 5 . Amplification $A$ versus inverse wavenumber $m^{-1}$ of compound jets with an inner to outer density ratio $\rho=0.5(a), 1(b)$ and $2(c)$. Numbers on the curves indicate the values of the inner to outer radius ratio $R$ (solid lines) and the inner to outer surface-tension ratio $\sigma$ (dashed lines). The shaded area in $(c)$ indicates the region in which breakup occurs by the meeting of the inner and outer interfaces.

In the following, we assume $C=1$, so that initial conditions are fixed only by the small parameter $\epsilon$. The inner-jet breaking time is reached when the minimum radius vanishes, $F_{\mathrm{m}}^{\mathrm{i}}=0$; in consequence

$$
t_{\mathrm{b}}^{\mathrm{i}}=\frac{1}{\tau} \ln \frac{R}{\epsilon A}
$$

On the other hand, there are two possibilities in calculating $t_{\mathrm{b}}^{\circ}$, depending on whether the outer interface reaches the inner one or not, as sketched in figure 4 . In the former case the breaking condition for the outer jet is $1-\epsilon\left(\exp \tau t_{\mathrm{b}}^{\circ}\right)=R-\epsilon A\left(\exp \tau t_{\mathrm{b}}^{0}\right)$, and the breaking time is

$$
t_{\mathrm{b}}^{o}=\frac{1}{\tau} \ln \frac{1-R}{\epsilon(1-A)},
$$

whereas in the second case the breaking condition is $F_{\mathrm{m}}^{\mathrm{o}}=0$ and then

$$
t_{\mathrm{b}}^{\circ}=\frac{1}{\tau} \ln \frac{1}{\epsilon} .
$$


The variation with $m^{-1}$ of the amplification $A$ corresponding to maximum growth factors $\tau_{\mathrm{m}}$ is shown in figure 5 . It must be stated that $A$ can be either positive or negative. According to (4.2), $A=0$ implies

$$
\tau^{2}=\frac{1}{2}\left(1-R^{2}\right)\left(m^{2}-m^{4}\right)
$$

the highest values of $\tau^{2}$ being obtained when $R=0$; hence

$$
\tau_{0}^{2}=\frac{1}{2}\left(m^{2}-m^{4}\right)
$$

This zero-amplification curve coincides with the upper boundary AC of the lower-branch root region (see figure 3 ). Points within this region give $A<0$, which means that outer and inner interface deformations are just in phase opposition. However, as already stated, lower-branch maxima are not significant, because they produce slower evolutions than upper-branch maxima.

As figure 5 shows, most of the compound-jet configurations have $A>1$. Innerinterface deformations are larger than outer-interface deformations, which seems to indicate that the inner jet will break before the outer jet. However, as $m^{-1}$ increases, $A$ decreases, in such a way that $A<1$ close to $m^{-1}=\sqrt{ } 2$. This means that inner-interface deformations are smaller than those of the outer interface, and could indicate that there is another breaking regime in which the outer interface reaches the inner interface before the minimum inner-interface radius vanishes. Some $A<1$ cases have a quite clear meaning. For instance, when $\rho=1, \sigma=0$ (single jet) the amplification is $A=R<1$ : fluid surfaces distort like the outer interface, the deformation being proportional to the undisturbed fluid-surface radius. When $\rho \neq 1$ the explanation is not so simple, and we should compare the breaking times of both inner and outer jets, $t_{\mathrm{b}}^{\mathrm{i}}$ and $t_{\mathrm{b}}^{\mathrm{o}}$ respectively. The outer interface will reach the inner interface when $t_{\mathrm{b}}^{\mathrm{i}}-t_{\mathrm{b}}^{\mathrm{o}}>0$, or, according to (4.4) and (4.6), $A / R=k, 0<k<1$; that is, $A=k R<1$. As shown in figure 5 , this breakup by interface meeting is possible if the breaking perturbation wavelength is large enough. Additional insight can be obtained from (4.2). After substituting $k R$ for $A$, we have

$$
\tau_{\mathrm{r}}^{2}=\frac{1}{2} \frac{1-R^{2}}{1-k R^{2}}\left(m^{2}-m^{4}\right)=\frac{1-R^{2}}{1-k R^{2}} \tau_{0}^{2}
$$

This expression gives, for each value of $m, R$ and $k$, the growth factor $\tau_{\mathbf{r}}$ for which a breakup by interface meeting would occur. $\tau_{\mathrm{r}}$ increases as $k$ increases, the highest values, $\tau_{\mathrm{r}}=\tau_{0}$, being obtained when $k=1(A=R)$. Therefore, if for a given wavenumber $m$ the maximum growth factor $\tau_{m}$ is smaller than $\tau_{0}$, breakup by interface meeting could occur. In contrast, if $\tau_{\mathrm{m}}>\tau_{0}$ the inner jet will break faster than the outer one. According to figure 3, the former breaking regime would only take place in the case $\rho>1$. In figure $5(c)$ the region of breakup by interface-meeting $(A \sim R)$ has been plotted.

\section{Experiments versus theory}

In order to evaluate the suitability of the one-dimensional model in predicting the behaviour of compound jets, theoretical results here obtained have been compared with experimental results reported by Hertz \& Hermanrud (1983). Two different compound jets are considered, in the first one a mixture of water $(80 \%)$ and glycerol $(20 \%)$ is used as outer liquid, and the same mixture, but dyed, as inner liquid. In the second compound jet the inner liquid is the same as in the first jet, whereas the outer fluid is a dimethyl silicone oil. 
For the first jet $(\sigma=0, \rho=1, R=0.488)$ theory predicts the same behaviour as the single jet: breaking should occur at the optimum $m^{-1}=\sqrt{ } 2$ (see table 1 ), which is in agreement with the value measured in figure 5 from Hertz \& Hermanrud (1983), $m^{-1}=1.4$. Furthermore, as can be seen from that figure, $A \sim R$ : inner-jet deformations are proportional to outer-jet deformations.

The second jet $(\sigma \sim 2.6, \rho \sim 1, R=0.488)$ seems to be more interesting for comparison purposes. In this case the corresponding theoretical value for the optimum $m^{-1}$ is 0.72 , and the values for $m^{-1}$ measured in figure 5 from Hertz \& Hermanrud (1983) are $m^{-1}=0.64$ (in the first wave) and $m^{-1}=0.60$ (mean value along the jet), close enough to theoretical value to consider the agreement between theory and experiments as significantly good, in spite of the errors involved in these estimations.

Concerning the amplification $A$, two factors mainly prevent us from making definitive conclusions on this point. The first one is the magnitude of the errors involved in making measurements from photographs shown in figure 6 from Hertz \& Hermanrud (1983), especially of the deformation of the outer interface, even if a cathetometer is used. The second factor concerns the lack of data on the outer-liquid refractive index, needed to correct the optical distortion of the inner interface. A value $A \approx 3$ could be guessed in the last two waves before inner-jet breaking (for which the linear model would not be suitable), the theoretical prediction for optimum breaking condition being $A=2.59$.

\section{Conclusions}

The behaviour of compound jets has been analysed by using a one-dimensional inviscid model which includes the main characteristics of such capillary jets. The influence of the parameters involved $(R, \rho, \sigma)$ has been studied through a linearized analysis, and, amongst other features, the existence of two breaking regimes should be pointed out. A better definition of these breaking regimes would require the extension of the analysis to nonlinear approximations of the model presented in $\S 2$, or even the numerical integration of the complete set of equations, but these tasks are outside the scope of this paper and should be undertaken in future work.

In addition, theoretical results have been compared with experimental ones, and, concerning the inverse wavenumber $m^{-1}$, the results here obtained agree with those of Hertz \& Hermanrud (1983). An interesting point for future experiments could be the exploration of jets lying in the coupled-breaking region (which the majority of the figures here deal with), and especially the interface-meeting region.

\section{REFERENCES}

BaUer, H. F. 1982 Coupled oscillations of a solidly rotating liquid bridge. Acta Astronautica 9 , $547-563$.

Bogy, D. B. 1978 Use of one-dimensional Cosserat theory to study instability in a viscous liquid jet. Phys. Fluids 21, 190-197.

Bogr, D. B. 1979 Drop formation in a circular liquid jet. Ann. Rev. Fluid Mech. 11, 207-228.

Bogy, D. B. 1981 Steady draw-down of a liquid jet under surface tension and gravity. J. Fluid Mech. 105, 157-176.

Chaudhary, K. C. \& Redekopp, L. C. 1980 The non-linear instability of a liquid jet. Part 1. Theory. J. Fluid Mech. 96, 257-274. 
Entov, V. M. \& Yarin, A. L. 1984 The dynamics of thin liquid jets in air. J. Fluid Mech. 140, 91-111.

Green, A. E. 1976 On the non-linear behaviour of fluid jets. Intl J. Engng Sci. 14, 49-63.

Hermanrud, B. 1981 The compound jet. A new method to generate fluid jets for ink printing. Rep. 1/1981, LUTEDX/(TEEM-1006)/1-143, Lund Inst. Tech., Sweden.

Hermanrud, B. \& Hertz, C. H. 1979 Ink jet development at the Lund Institute of Technology. J. Appl. Photogr. Engng 5, 220-225.

Hertz, C. H. \& Hermanrud, B. 1983 A liquid compound jet. J. Fluid Mech. 131, 271-287.

Keller, J. B., Rubinow, S. I. \& Tu, Y. O. 1973 Spatial instability of a jet. Phys. Fluids 16, $2052-2055$.

LEE, H. C. 1974 Drop formation in a liquid jet. IBM J. Res. Dev. 18, 364-369.

MesegVer, J. 1983 The breaking of axisymmetric liquid bridges. J. Fluid Mech. 130, 123-151.

Meseguer, J. \& Sanz, A. 1985 Numerical and experimental study of the dynamies of axisymmetric liquid bridges. J. Fluid Mech. 153, 83-101.

Pimbley, W. T. \& Lee, H. C. 1977 Satellite droplet formation in a liquid jet. IBM J. Res. Dev. 21, 21-30.

Sanz, A. 1983 Comportamiento de las zonas liquidas flotantes en microgravedad simulada. Tesis doctoral, Universidad Politécnica de Madrid.

Schlichting, H. 1960 Boundary Layer Theory, chap. Xi. MeGraw-Hill

Томотіка, S. 1935 On the instability of a cylindrical thread of a viscous liquid surrounded by another viscous fluid. Proc. R. Soc. Lond. A 150, 322-337.

Weber, C. 1931 Zum Zerfall eines Flüssigkeitsstrahles. Z. angew. Math. Mech. 11, 136-141. 